\title{
Common Pitfalls in Communications Systems Simulation
}

\section{Dr. Miguel Bazdresch, Rochester Institute of Technology (CAST)}

Miguel Bazdresch obtained his $\mathrm{PhD}$ in Electronic Communications from the Ecole Nationale Superieure des Telecommunications, in France, in 2004. He worked for several years designing digital integrated circuits for the telecommunications industry. After teaching at ITESO University, in Mexico, from 2005 to 2012, he came to the Electronics, Computers and Telecommunications Engineering Technology Department at the Rochester Institute of Technology, where he is an Assistant Professor. 


\title{
Common Pitfalls in Communications Systems Simulation
}

\begin{abstract}
In this paper, we present some common pitfalls students face when simulating communications systems. Over the years, we have found that students consistently make the same mistakes and have difficulty grasping and mastering the same concepts. This presents an excellent opportunity to develop teaching and learning strategies that address these specific pitfalls, especially given that they form a relatively small set. We focus on techniques related to communications and signals concepts, not on the details of operating a particular simulation tool. Along with more detailed descriptions of common pitfalls, we present some teaching and learning strategies we have developed to address them.
\end{abstract}

\section{Introduction}

Simulation plays an important role in engineering education, ${ }^{1}$ and communications systems education is not the exception. ${ }^{2}$ Using a numerical computing language, students can put in practice the theory behind communications and signals and systems, easily plot signals and their spectra, and predict the performance of eventual hardware implementations. Simulation, especially when coupled with a dynamic, scripting language like Matlab or Python, is also a very good environment for learning and practicing skills such as system debugging and performance evaluation.

A course on communications systems can be organized in myriad ways, and many approaches have been reported in the discipline-based education research literature. One possibility is to restrict contents to theory. A course can also cover system theory and use simulation as a tool to enhance student understanding and to promote exploration in system design. There are wellregarded textbooks that support this approach. ${ }^{3,4}$

It is also possible to use a numerical language as the basis of hands-on experimentation. Very low-cost approaches, based on using a computer's soundcard as an analog front-end, are feasible. ${ }^{5}$ Other reported approaches digitize laboratory-generated signals, which are later processed and analyzed by students. ${ }^{6}$

Simulation software can be used at different levels of complexity. For example, block-diagram simulations where the user only modifies system parameters and obtains visual results can be used when appropriate for the given intended learning outcomes. ${ }^{7,8}$

Student difficulties with simulation software have been reported and addressed using special courseware. ${ }^{9}$ We identify particular pitfalls and try to solve them without any additional coursework.

Other approaches that have been reported include using symbolic mathematical software (such as Mathcad) instead of numerical computing software (such as Matlab). ${ }^{10}$ Such software is used 
mainly to generate plots and spectra of equations. Also, LabView ${ }^{11}$, Simulink $^{12}$ and SystemVue ${ }^{13}$ are used to connect blocks, making it easy to set up pre-defined experiments.

We focus on simulation as an intermediate step between theory and practice, as part of an engineering technology communications curriculum. The purpose of simulation in this scenario is both to improve students' grasp of the theoretical foundations of communications, and to get them started in the exploration of different implementation strategies. At the same time, students can get a "feel" for what to expect, performance-wise, from their implementation. Plots, spectra and other results obtained in simulation let students know how their implementation should behave; in this sense, it aids both in debugging and in performance evaluation.

To illustrate the intended learning process, consider this specific example. The instructor sets up a learning scenario where students will implement, test and evaluate an AM system. Two teams send their AM-modulated voices over different channels, and two other teams receive the voice signals. Students would start by stating the problem in mathematical terms, supported by Fourier theory. In this domain, signals (their voices) are just mathematics written on a piece of paper. Eventually, they figure out the equations; the system, being ideal, works perfectly.

In the second step, students would implement the system in a numerical computing package, such as Matlab. The choice of package, and at what abstraction level to set up the simulation, has an effect on the time students will require for this task, but also on the depth of their understanding. This aspect is explored in depth in many of the references given above. We have chosen to use Matlab; we ask students to implement the system at a fairly low-level and with few, if any, pre-made, "black-box" blocks. Whatever the choice of numerical package, the simulation tool allows the students to build and test their system, since they may use actual signals, for voice, carriers, etcetera. They will find that the system's performance is different from that predicted by theory - no filter has infinite rejection, for example.

The final step is to actually build the AM communications system, using analog circuitry. (This process may also be aided by simulation of a different kind: analog circuit simulation). The system's performance is, again, different from that calculated in simulation. However, results obtained in both theory and simulation aid the students when building the system, since they know what to expect at every step.

Properly setting up this learning scenario is not trivial. In addition, numerical simulation is discrete by its nature, which presents a disconnect from both Fourier theory (as the students most probably know it) and the circuitry implementation, which are analog. This disconnect presents difficulties, whose solutions are the focus of this paper. Our aim is to provide a head start to instructors introducing numerical simulation in their courses, and share ideas with instructors already experienced in this area. 


\section{Description of Common Pitfalls}

In this section, we present six problems that students very commonly have when first learning how to simulate a communications system in a numerical simulation tool. We describe the problems, from our perspective as instructors, and then present exercises that we have found help students to quickly grasp the concepts involved.

Representing an analog signal as discrete. Even for students who have already used a numerical tool for numerical calculations, it can be difficult to achieve fluency in dealing with discrete signals. Since electrical systems in general, and communications systems in particular, have analog inputs and outputs, moving to the digital domain may seem an unnecessary complication. In our experience, students face two main roadblocks.

First, in Matlab and similar packages, a signal is just a vector. There is no intrinsic concept of time. The user must interpret the vector elements as samples taken at time instants that are not always indicated. One method to get students used to the idea that discrete signals and vectors are essentially the same thing is to describe a signal as two vectors, not one. One is a vector of samples, and the other is a vector of time instants. Consider the following exercise:

- Exercise: Generate two signals, each a cosine of different frequency, using an appropriate sampling frequency. Keep track of both samples and time instants for both signals. Do the following operations on the signals:

○ Add and multiply the signals. What is the time vector that corresponds to the result?

- Calculate the output of a linear, time-invariant system whose input is one of your signals. Remember to calculate both the samples and time instants of the output.

- Resample the signals to increase/decrease their sampling rate by a factor of 2 . Recalculate the time instants.

In the second place, students may be reluctant to learn the subtleties involved in digital signal processing. It is motivational to propose an exercise that showcases how straightforward it is to generate interesting effects on a signal, compared to doing the same in analog circuitry:

- Exercise: Plug a microphone and loudspeakers to your computer. Record a few seconds of your voice, at a sampling rate of 16 kilosamples per second (ksps). Create code to perform the following experiments:

- Plot the voice samples you captured.

- Plot a spectrogram of the samples.

- Reproduce the samples on the loudspeakers. Is the signal coming from the speakers discrete or continuous?

- Reproduce the voice at sampling rates higher and slower than that used for capturing. How are the signals different/similar when the sampling rate changes? 
$\circ$ Use low-pass, band-pass and high-pass filters on the signal. How would you describe their effects on the voice?

Other effects (echo, distortion) could be proposed to more ambitious students. Note that signal processing (at least in some simulation tools) can even be done in real time, making the experiment more interesting. ${ }^{5}$
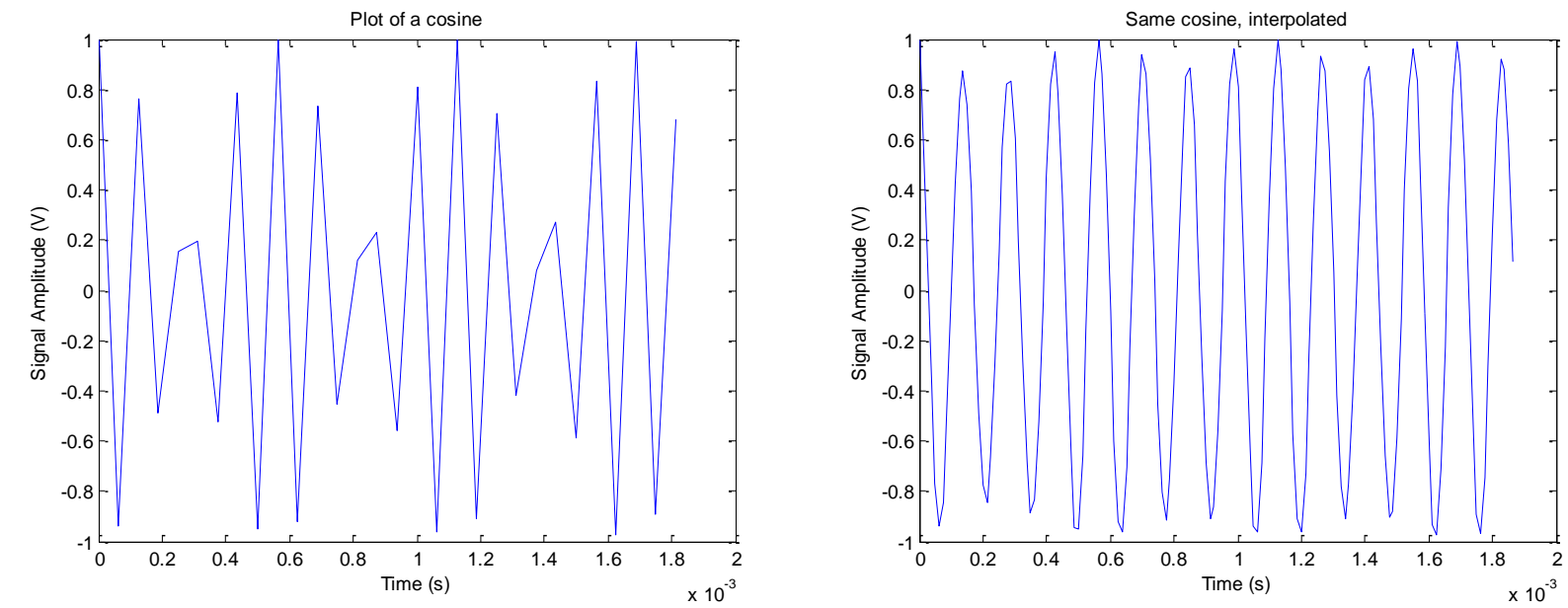

Figure 1. On the left, a cosine signal sampled at close to the Nyquist rate. On the right, the same signal, upsampled five times.

Sampling and plotting. When studying a signal on an oscilloscope, one expects to see the actual waveform displayed. The same is not necessarily the case in simulation. Students learn that, if a signal is sampled at a rate at least twice its maximum frequency, then all the information about the signal is captured in its samples. However, this does not mean that the signal may be plotted by straightforward means. When the sampling frequency is close to its minimum value, plotting the signal does not result in a waveform similar to what could be expected by a beginner. This is illustrated in Fig. 1.

Students who insist on "smooth" signal plots may seek to use a higher sampling frequency. This must be discouraged, because it may have negative effects on the simulation.

Two exercises to help students grasp that a vector actually contains a given signal even if its plot seems wrong are the following:

- Exercise: Generate a $7.1 \mathrm{kHz}$ cosine signal sampled at 16 kilosamples per second (ksps). Plot a few periods of the signal. Output the signal through the computer's sound card. Listen to it through loudspeakers, and also observe it in an oscilloscope. Repeat the process for a sampling rate of $48 \mathrm{ksps}$. Verify that both signals actually have the same shape and properties, even if their computer plots are very different. (Note that the sampling rates have been chosen to be compatible with most modern sound cards). 
- Exercise: Generate a $7.1 \mathrm{kHz}$ cosine signal sampled at $16 \mathrm{ksps}$. Plot a few periods of the signal. Interpolate the signal to increase its sampling rate 20 times, and plot it again. (Note: Unless care is taken, this method may be confusing to the students, since the filtering involved in the interpolation process changes the signal duration, and the interpolation does not reconstruct the signal perfectly).

The Nyquist Frequency. Students' difficulties with fully grasping the concept of Nyquist frequency are two-folded. First, they need to calculate the appropriate Nyquist frequency for their simulation, by determining the maximum frequency that will appear at any point in the system. Second, they need to understand the folding that occurs around the Nyquist frequency, and avoid any undesired effects caused by it.

We can illustrate these difficulties using a simulation of AM broadcasting as an example. In this exercise, students transmit and receive a number of signals using double side-band, large carrier amplitude modulation. The mathematical formulation is as follows. Assuming three signals to transmit, $s_{1}(t), s_{2}(t)$, and $s_{3}(t)$, each with amplitude in $[-1,1]$ and with bandwidth $B=$ $10 \mathrm{kHz}$, modulation level $50 \%$, and three carrier frequencies $f_{1}=30 \mathrm{kHz}, f_{2}=60 \mathrm{kHz}$, and $f_{3}(t)=90 \mathrm{kHz}$, then the received signal is

$$
r(t)=\left(1.5+s_{1}(t)\right) \cos \left(2 \pi f_{1} t\right)+\left(1.5+s_{2}(t)\right) \cos \left(2 \pi f_{2} t\right)+\left(1.5+s_{3}(t)\right) \cos \left(2 \pi f_{3} t\right) .
$$

From the received signal, recovering $s_{i}(t)$ involves the following steps:

- Filter $r(t)$ with a band-pass filter of bandwidth $20 \mathrm{kHz}$ and centered around $f_{i}$.

- Input the filtered signal to an envelope detector (a non-linear element followed by a lowpass filter of cutoff frequency of $10 \mathrm{kHz}$ ).

- Remove the DC component from the low-pass filter's output.

(The process can be done differently, but this is arguably the simplest formulation). Once the mathematics behind this process are understood, students proceed to implement the system in Matlab. In doing so, they face the following questions:

What is the required sampling rate? When analyzing the transmitter, the most common mistake is using the highest carrier frequency $\left(f_{s}>120 \mathrm{kHz}\right.$, which is erroneous), without accounting for the message's bandwidth $\left(f_{s}>140 \mathrm{kHz}\right.$, which is correct).

The receiver, however, poses a much harder problem. Since the envelope detector is non-linear, its output is difficult to analyze and, in consequence, it is also difficult to determine the sampling rate. From the instructor's perspective, some possible alternatives to deal with this problem are:

- Instruct students to use a very high sampling rate (several times the minimum required), without further explanation (this is the approach taken by some textbooks ${ }^{3}$ ). In this approach, the demodulated signal appears in the baseband by some unexplained process. 
- Specify a very high sampling rate, but analyze the signal's harmonics at the output of the non-linear element. Even without a detailed understanding of the origin of all the observed harmonics, the spectrum may be useful to determine an appropriate sampling frequency.

- Use the power-series expansion of the non-linear element to explain intermodulation. Single-tone modulation may be a useful simplification. As an example, if the non-linear element is the absolute value operation and its input is $u(t)$, then

$$
\begin{aligned}
|u(t)| & =\left|\left(1.5+s_{i}(t)\right) \cos \left(2 \pi f_{i} t\right)\right| \\
& =C+\alpha_{1}\left(u^{2}(t)+\beta_{1}(t)\right)+\alpha_{2}\left(u^{4}(t)+\beta_{2}(t)\right)+\alpha_{3}\left(u^{6}(t)+\beta_{3}(t)\right)+\cdots
\end{aligned}
$$

From here, a bit of algebra and trigonometry reveals the reason why the demodulated signal is present in baseband, and also the origin of all harmonics. Students can also get familiar with the concept of intermodulation distortion.

In all cases, the sampling frequency must be chosen with care. The reason is that the harmonics beyond the Nyquist frequency will be folded back into the spectrum range, potentially interfering with the demodulated message. For example, consider transmission of a $10 \mathrm{~Hz}$ single-tone signal with a $50 \mathrm{~Hz}$ carrier. Choosing a sampling frequency $f_{s}=405 \mathrm{~Hz}$, an undesired harmonic will be folded back to a frequency of $5 \mathrm{~Hz}$, causing undesired (and, to the student, unexpected) interference.

In our experience, around a third of students end up choosing an incorrect sampling frequency either too low, or a value that results in interference because of folded harmonics.

Resampling. After determining the highest frequency that will appear in a simulation, the simplest way to set up the simulation is to resample all input signals to match the required sampling signal. This is especially true in a system with several inputs and several outputs, each of them with possibly a different sampling rate. Consider the following exercise:

- Exercise: Simulate an AM DSB-LC system with three audio inputs. The inputs have been sampled at rates 8000, 16000 and $32000 \mathrm{ksps}$, respectively. Each AM channel has a bandwidth of $30 \mathrm{kHz}$ and the maximum carrier frequency is $150 \mathrm{kHz}$. The receivers use an envelope detector and output the demodulated signal to a sound system that requires a sampling rate of $48000 \mathrm{ksps}$. Find the sampling frequency at which the simulation will run, and calculate the resampling ratios for each signal.

Diagnose system behavior. Simulation can be a powerful tool to discover errors in a communications system design. However, students need to be taught how to recognize errors and how to find their root causes. ${ }^{14}$ In our experience, it is too common that students, when they obtain sub-optimal performance from their systems, try to find justifications or simple explanations, instead of spending the time and effort to discover their mistakes. It is possible, 
however, to instruct them in proper debugging techniques so that the time and effort is wellguided.

Here, we focus on one particular pitfall: relying too much on spectrum analysis to debug errors. Once students become comfortable calculating and interpreting signals' spectra, they may forget that time analysis can sometimes be more useful. Consider this exercise:

- Exercise: Consider an AM double side-band, suppressed carrier system. The (noncoherent) carrier at the receiver has an offset of $0.5 \mathrm{~Hz}$ with respect to the transmitter carrier frequency. Simulate this system and verify that theory's predictions are correct.

Trying to solve this problem using frequency analysis is futile; the spectra of the received signal with and without offset are practically indistinguishable. However, a plot in time immediately reveals the problem introduced by frequency offset.

Filter design. One of the main advantages of digital signal processing is the ease with which filters can be designed and applied.

Since the purpose of a communications course is not to go into the intricacies of the different digital filter architectures and design algorithms, it is possible to take a "black box" approach and use automated filter design commands, such as Matlab's fir2. There is no need to use more complex architectures than finite impulse response filters. Filter order is not a concern, since modern computers are not slowed down by a few million arithmetic operations and efficiency is not one of our goals.

Having said this, students usually struggle with a few of the concepts and techniques involved.

How to tell a good filter from a bad filter? When implementing a communications system in a numerical tool, students will spend much of their time designing low-pass, band-pass and highpass filters. However, students tend to trust the numerical tools too much, and do not stop to evaluate the filter response that the tool is creating for them. A filter may be deficient for one of two reasons:

- Not enough out-of-band rejection. This is by far the most common problem. In most cases, students should aim for at least $30-$ to $40-\mathrm{dB}$ rejection.

- Non-flat response in the pass-band. This is especially problematic with very narrow filters. However, the definition of "narrow" is relative to the Nyquist frequency - a filter of the same width will be easier to design with a smaller Nyquist frequency. This interplays with the choice of Nyquist frequency; choosing too high a sampling rate results in difficulties when designing filters.

Moreover, at what point a particular filter response is determined to be "good" is subjective and could be said to be an engineering decision, since it depends on performance criteria set 
beforehand. Asking students to judge their filters and decide when they meet their requirements is good practice for them.

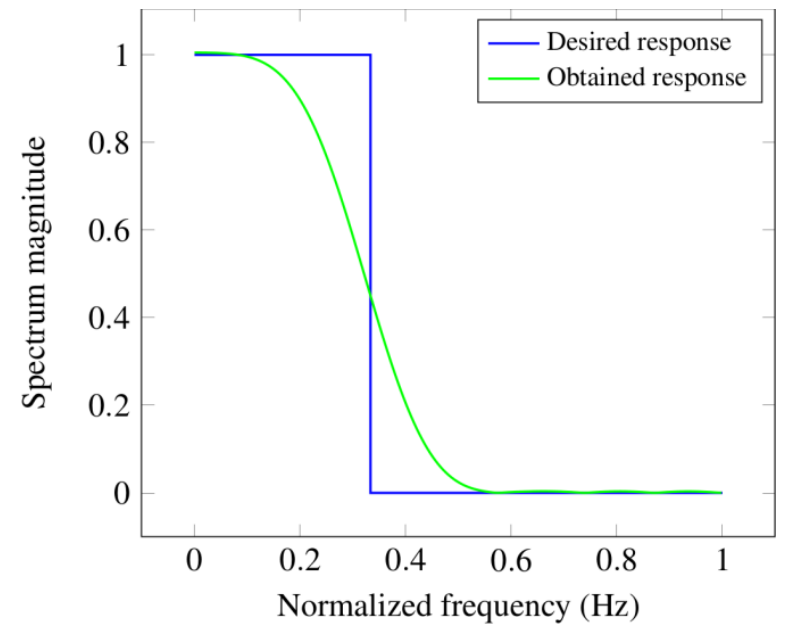

(a) Filter order 16.

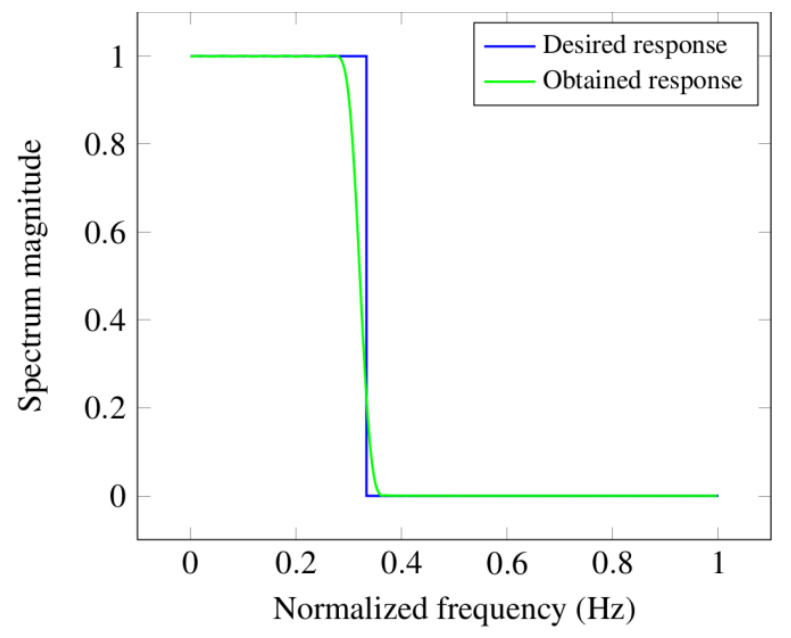

(b) Filter order 100.

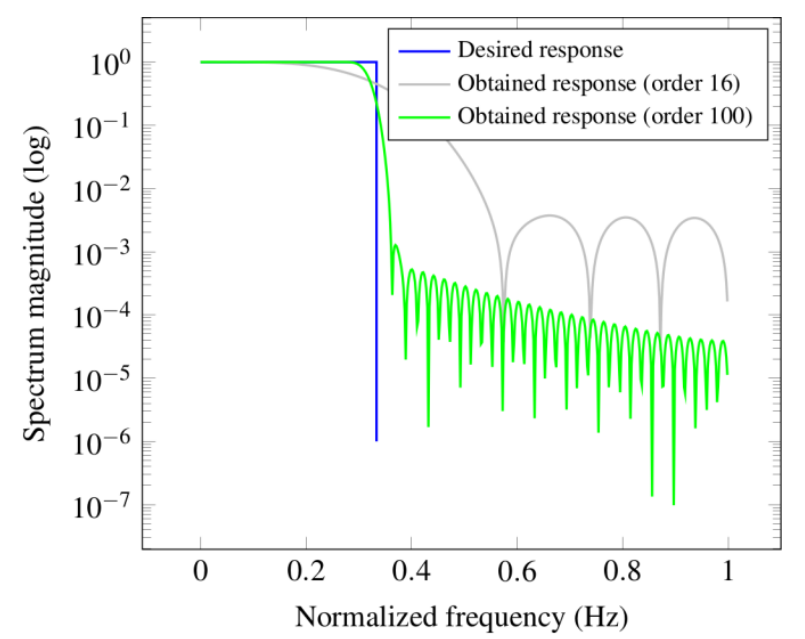

(c) Logarithmic plot, showing a rejection of approximately $10^{-3}$ for an order-16 filter, and $10^{-4}$ for a 100 -order filter.

Figure 2. Plots of desired and obtained filter responses. The filters were designed using Matlab's fir2 command.

How to choose the filter order? Increasing the filter order is the most straightforward way to try to improve on a filter's response. However, most filter design algorithms (such as Remez) exhibit diminishing returns when the order increases too much. Even though the purpose is not to find the most efficient filter, it makes sense to ask students to use the lowest-order filter that meets their needs. The following exercises help students learn about these issues. 
- Exercise: Generate a signal composed of the addition of two sine waves of frequencies $f_{1}=1000 \mathrm{~Hz}$ and $f_{2}=5000 \mathrm{~Hz}$. Design a low-pass filter that eliminates the $5000 \mathrm{~Hz}$ signal. Follow these steps:

- Plot the signal, its spectrum, and listen to it. Identify both cosine frequencies.

- Implement a 16-order low-pass filter to reject the $5000 \mathrm{~Hz}$ cosine. Plot the filter's ideal response versus the actual response (see Fig. 2a).

- Filter the signal. Plot the result, its spectrum, and listen to it. Is the 16-order filter good?

○ Repeat for a 100-order filter (see Fig. 2b). Is this filter good? Is it better or worse than the 16 -order filter?

Plotting the desired (ideal) and the actual obtained filter responses on the same plot is an excellent way to analyze the filters. Note, however, that in Figs. $2 \mathrm{a}$ and $2 \mathrm{~b}$ it is impossible to tell what the actual rejection is. Using a logarithmic scale is more illustrative (see Fig. 2c).

- Exercise: Plot the filter's responses using a logarithmic scale for the magnitude.

How to improve the filter's response? Besides increasing the order, it can be done by relaxing the slope at the transitions. When increasing the order fails, the best alternative is to relax the filter requirements. This is especially true for band-pass and for very narrow filters. Students need to experiment with the slopes at transitions, until they find a combination that meets their system requirements.

\section{Assessment}

We have presented a summary of observations made during many years of teaching communications courses with a simulation component, and we have proposed exercises that, in our experience, are helpful to students. We can indirectly assess their efficiency by observing the learning and results obtained by students in a course. In a 15-week, 1-credit laboratory course, students have been able to fully simulate, build, and test, a two-channel AM system (transmitter and receiver). Students didn't have any numerical computing experience at the start of the course. Moreover, it has been observed that simulation results provide a set of known-good, expected results, which makes debugging analog circuitry much easier.

\section{Conclusions}

After several years of teaching communications courses with a simulation component, we have developed a list of common pitfalls that students face, as well as exercises designed to help students avoid them and learn in the most efficient manner. We have presented a summary of these observations in this paper. We believe that simulation will continue to play an increasingly important role in engineering and in electrical communications education. The teaching community should continue to spend time and effort in developing increasingly efficient 
methods to help students profit from this tool both in their engineering education and in their professional life.

\section{Bibliography}

1. Gokhale A., "Effectiveness of Computer Simulation for Enhancing Higher Order Thinking", Journal of Industrial Teacher Education, Vol. 33, No. 4, 1996.

2. Tranter W. H. et al, "The Role of Simulation in the Teaching of Communications", Proc. of Frontiers in Education Conference, 1996, Vol. 1, pp. 401-404, DOI: 10.1109/FIE.1996.569995.

3. Johnson, C. R. et al, "Software Receiver Design: Build Your Own Digital Communication System in Five Easy Steps", Cambridge University Press, September 2011.

4. Silage, D., "Digital Communication Systems using MATLAB and Simulink", Bookstand Publishing, August 2009.

5. Bazdresch, M., "A Real-Time, Matlab-Based Undergraduate Digital Communications Course," Digital Signal Processing Workshop and IEEE Signal Processing Education Workshop (DSP/SPE), Sedona, AZ, USA, pp. 408-413, 4-7 Jan. 2011, DOI: 10.1109/DSP-SPE.2011.5739249

6. Zhang J. et al, "Theory, Practice and Systems-A new Approach to Teaching Electronic Communications with Matlab", Int. J. Engng Ed., Vol. 21, No. 4., pp. 612-617, 2005.

7. Frolik J., "Laboratory Enhancement of Digital and Wireless Communications Courses", Proc. of the ASEE 2005 Annual Conference, Portland, OR, USA, June 2005.

8. Frolik J., "A Comprehensive, Laboratory-Enhanced Communications Curriculum”, Proc. of the ASEE 2004 Annual Conference.

9. Kramer K. et al, "Demonstrating Complex Communication Systems Principles Using Electronic Courseware and a Simple Computer Math Package", Proc. of the ASEE 1998 Annual Conference.

10. Border B., "Integration of National Instruments Multisim and Mathsoft Mathcad into a Digital Communications Technology Curriculum", Proc. of the ASEE 2012 Annual Conference.

11. Doering E. and Shearman S., "Communication Systems Laboratory Projects Featuring Interactive Simulation and Visualization", Proc. of the ASEE 2009 Annual Conference.

12. Li X. et al, "Simulink-Based Simulation Of Quadrature Amplitude Modulation System", Proc. of the 2007 ASEE Annual Conference.

13. D. Silage, "Teaching Digital Communications in a Wireless World: Who Needs Equations?", Proc. of the ASEE 2006 Annual Conference.

14. Steffen G., "Learning Through Error Recognition Using The Three Strikes Method", Proc. of the ASEE 2008 Annual Conference. 